\title{
Development of a Real-Time Fuel Processor Model for HIL Simula- tion
}

\author{
Karin Fröjd ${ }^{1}$, Karin Axelsson ${ }^{2}$, Ivar Torstensson ${ }^{1}$, Erik Åberg ${ }^{1}$, Erik Osvaldsson ${ }^{2}$, Gregor Dolanc ${ }^{3}$, \\ Bostjan Pregelj ${ }^{3}$, Jonas Eborn ${ }^{1}$, Jens Pålsson ${ }^{1}$ \\ ${ }^{1}$ Modelon AB, Ideon Science Park, Scheelev. 17, 22370 Lund, Sweden \\ ${ }^{2}$ PowerCell AB, Ruskvädersgatan 12, 41834 Göteborg, Sweden \\ ${ }^{3}$ J. Stefan Institute, Jamova cesta 39, 1000 Ljubljana, Slovenia
}

$\underline{\text { Karin.Frojd@modelon.com, Karin.Axelsson@powercell.se }}$

\begin{abstract}
In this article a real-time model for dynamic simulation of a fuel processor is presented. The model is intended for HIL testing of the PLC for a truck Auxiliary Power Unit (APU) system.

The APU comprises a PEM fuel cell and fuel processor to enable direct utilization of on-board diesel. The system is under development in FCGEN, an EU project under the FP7 program FCH JU [1]. One critical challenge is to design the control system (PLC) to ensure failsafe and environmental friendly startup and operation. The startup phase of the fuel processor is the most critical part, since it is a highly dynamic process involving several complex reactors. It is advantageous to verify the control system before the fuel processor is assembled to avoid possible breakage of components. Such verification can be done with a real-time model representing the physical system. In this study such a model is created using Modelica and Dymola. It is shown that it is possible to load and execute a real-time Modelica model capable of realistically mimicking the system response on a HIL platform. The model runs in real time using a first order explicit (Euler) solver with a time step size of $25 \mathrm{~ms}$.
\end{abstract}

Keywords: HIL simulation; fuel reformation; fuel processor, fuel cell, PLC, real-time simulation

\section{Abbreviations}

APU Auxiliary Power Unit

ATR Auto-Thermal Reformer

BoP Balance of Plant

CAB Catalytic After Burner

CSB Catalytic Start Burner

$\begin{array}{ll}\text { DS } & \text { Desulphurizer } \\ \text { FPM } & \text { Fuel Processing Module } \\ \text { HIL } & \text { Hardware In the Loop } \\ \text { PEM } & \text { Proton Exchange Membrane } \\ \text { PLC } & \text { Programmable Logic Controller } \\ \text { PrOx } & \text { Preferential Oxidation } \\ \text { WGS } & \text { Water Gas Shift }\end{array}$

\section{Introduction}

Fuel cell systems are an attractive technology for Auxiliary Power Units for e.g. trucks, because of the high efficiency and low emissions. However, PEM fuel cells operate most efficiently with hydrogen, while at gas stations usually only liquid fuels like diesel and gasoline are available. To circumvent this problem a fuel processor may be used to convert the high order hydrocarbons to a hydrogen rich gas mixture. The aim of the FCGEN [1] program is to design and demonstrate such an APU.

The fuel processor is a complicated system of reactors, heat exchangers and BoP components. System startup needs to be carefully designed to avoid poisoning of reactors and system failure and ensure as short startup time as possible. The control system must be designed to give a smooth and secure startup. Verification and adjustment of the control system is normally performed against the real system. However since the system is complex, such testing may become very expensive and time consuming. Hence it is advantageous to test the system against a model in a HIL (Hardware-In-the-Loop) setup before assembling the full system. In this work such a model is developed and tested. 
An example of a previous related work is a physical model made in Modelica of a food processor line tested in a HIL set up in 2008 [2].

\section{Fuel processor}

The fuel processor module (FPM) is based on an Auto-Thermal Reformer (ATR) which converts diesel to syngas through steam reformation. Required heat for the endothermic reformation reaction is supplied by oxidation with a limited amount of air.

A desulphurizer (DS) is added downstream of the ATR to remove sulphur to protect downstream catalysts and the fuel cell.

The PEM fuel cell tolerates only very low CO concentrations (ppm levels). To remove $\mathrm{CO}$ from the syngas to the fuel cell, a water-gas shift (WGS) reactor and a preferential oxidizer (PrOx) are added downstream of the DS. To avoid poisoning of the fuel cell during the startup phase, a bypass route is used. A catalytic afterburner $(\mathrm{CAB})$ is used to clean the exhaust gases before release to the atmosphere. In the early start phase a start system is in operation burning diesel in a catalytic start burner (CSB) to pre-heat the FPM. The system scheme is shown in Fig. 1 below.

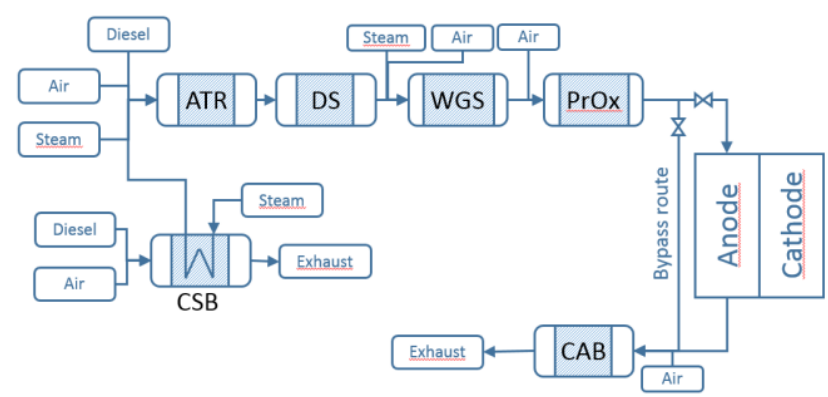

Figure 1: Fuel processor system scheme

The fuel processing module (FPM) is developed by the following partners of FCGEN:

- Volvo Group Trucks Technology, Sweden coordinator

- PowerCell Sweden AB - plant components, system specification, integration and testing

- Forschungszentrum Jülich GmbH, Germany $\mathrm{ATR}$ and $\mathrm{CAB}$ reactors

- Institut für Mikrotechnik Mainz GmbH, Germany - DS, WGS and ProX reactors

- Johnson Matthey PLC, UK - catalysts for reactors

- Jozef Stefan Institute, Slovenia - control system

- Modelon AB, Sweden - dynamic system model

\subsection{Startup strategy}

The main steps of the startup strategy are;

1. Use the start burner to heat the ATR and downstream reactors to sufficient temperature.

2. Use the start burner to produce steam for the ATR.

3. Ignite the ATR, keep start burner in operation to ensure sufficient fuel and emission conversion.

4. Shut down start burner, start normal operation.

5. Stop by-pass of fuel cell.

\section{Requirements on HIL model}

The aim of this work is to create a model that can be used to test the PLC logic. The simplifications needed to meet the real-time requirement sets a limit on model accuracy. Hence the model will not be suitable nor be used for calibration of PLC logic parameters. The requirements of the HIL simulation model are defined as:

1. The model should be robust and never crash.

2. The PLC requires real-time communication with the plant model. Consequently, no solver step may require a CPU time longer than real time (so called overrun).

3. The model should respond realistically to changes in input signals. For example ATR temperature should respond in the right direction when changing air-fuel ratio or steam mass flow.

4. Trends need to be captured for temperatures and mass flows. Exact numbers are not necessary.

5. Response times are allowed to deviate slightly from reality. In general model response times are longer than real response times, because of the constraint on minimum time constants.

To meet requirement no 2 the model must be fairly simple, and an explicit solver such as the first order Euler method need to be applied. Fast dynamic time scales need to be removed by changing dynamic equations to static. Events need to be eliminated to avoid solver reinitialisation and subsequent overruns. At the same time the model needs to respond realistically to signal changes, as defined in requirement no 3 . However, because of the simplicity of the model it cannot be expected to correctly predict absolute temperatures 
or times of the system. This accuracy is not needed for the PLC tests.

\section{Model description}

The Modelica model is based on the Fuel Cell Library from Modelon [3]. This newly released product contains a number of component models and examples aimed at PEMFC and other fuel cell applications including fuel processing reactors. The library depends on Modelon Base Library for base classes common to several Modelon libraries. Early work and cornerstone of FCL was carried out by Andersson and Åberg [4]. Dymola features such as drag and drop functionality, graphical user interface, equations in text layer and numerical solvers facilitate development of a dynamic fuel cell model.

The full system includes more than 25 components, including compressors, heat exchangers, valves, reactors and pumps. All relevant components of the fuel processor have been included in the model. The realtime model comprises 217 continuous time states and is of index 1 .

Two-phase water medium has been used where necessary. For model robustness pure steam medium was applied wherever possible. Multi-component gas phase media was used for reformate gas and air.

To enable HIL simulations and meet real-time requirement, all extended component models (submodels) need to be simple.

The Dymola model is initially developed for the DASSL implicit ODE solver. To avoid overruns in the HIL simulations an explicit fixed-step solver must be used. The first order Euler solver is applied in this work. To match the real time requirement a sufficiently large time step size need to be used in the Euler solver.

In the following sections the main submodels, realtime adaptations and HIL setup are described.

\subsection{Chemical reactors}

Chemistry is very complex and the final composition is highly dependent on the initial state and local phenomena. Simplified chemistry models are usually valid in a narrow state space. The purpose of this study is to validate and test the FPM control system during normal system startup. It is enough to predict reasonable heat release in each reactor. Detailed species concentrations are not required. Hence simple chemistry models designed for the normal startup and operation range are suitable.
In the ATR fuel is reformed by steam under presence of a limited amount of oxygen. Fuel lean combustion does not need to be covered by the model, and complete conversion is a valid assumption.

The reactors are implemented as homogeneous (lumped) reactor models, utilizing different reaction objects to simulate reaction time characteristics typical for each reactor. The reaction objects used are equilibrium chemistry, complete conversion and reaction kinetics using Arrhenius equation. Heat exchangers, flow losses and heat losses are included where applicable. See Fig. 2 for Dymola model schematics of the WGS reactor. The following chemical reactions are applied in the system:

Combustion (ATR):

$$
\mathrm{C}_{n} \mathrm{H}_{m}+\left(n+\frac{m}{4}\right) \mathrm{O}_{2} \rightarrow n \mathrm{CO}_{2}+\frac{m}{2} \mathrm{H}_{2} \mathrm{O}
$$

Partial oxidation (ATR):

$$
\mathrm{C}_{n} \mathrm{H}_{m}+\frac{n}{2} \mathrm{O}_{2} \rightarrow \mathrm{nCO}+\frac{m}{2} \mathrm{H}_{2}
$$

Hydrocarbon steam reformation (ATR):

$$
\mathrm{C}_{n} \mathrm{H}_{m}+n \mathrm{H}_{2} \mathrm{O} \rightarrow n \mathrm{CO}+\left(n+\frac{m}{2}\right) \mathrm{H}_{2}
$$

Water-gas shift (ATR, WGS):

$$
\mathrm{CO}+\mathrm{H}_{2} \mathrm{O} \leftrightarrow \mathrm{CO}_{2}+\mathrm{H}_{2}
$$

$\mathrm{H}_{2}$ oxidation ( $\left.\mathrm{PrOx}, \mathrm{CAB}\right)$ :

$$
2 \mathrm{H}_{2}+\mathrm{O}_{2} \leftrightarrow 2 \mathrm{H}_{2} \mathrm{O}
$$

$\mathrm{CO}$ oxidation (PrOx, $\mathrm{CAB})$ :

$$
2 \mathrm{CO}+\mathrm{O}_{2} \leftrightarrow 2 \mathrm{CO}_{2}
$$

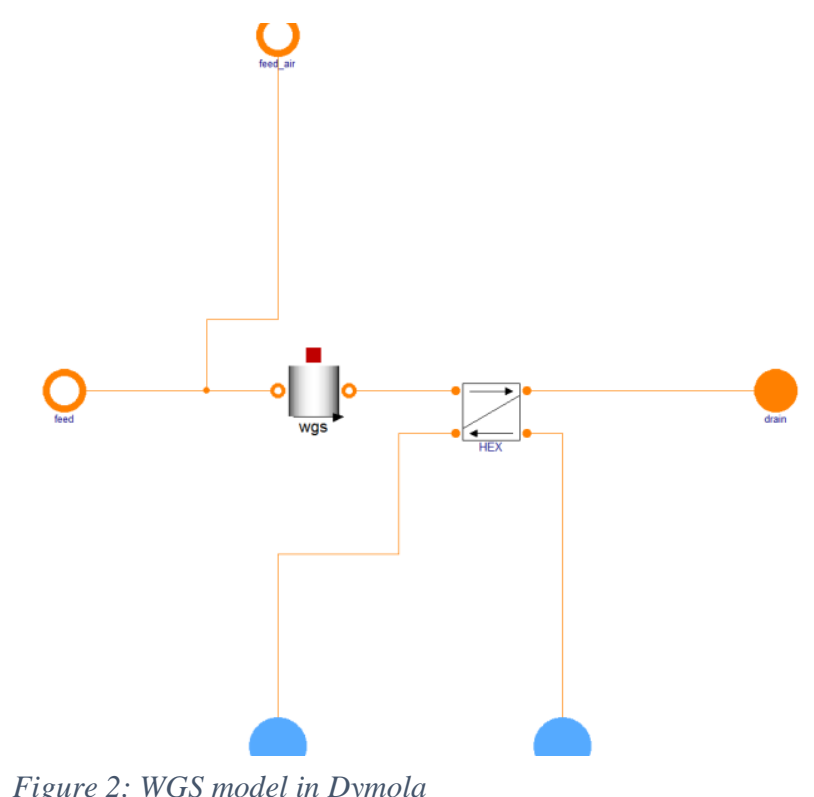

Figure 2: WGS model in Dymola

\subsection{Start system}

The catalytic start burner from Zemission is simply modeled by tabulated temperatures and mass flows as 
function of power load. The table based start burner is connected to heat exchangers transferring the heat to the rest of the fuel processor (see Fig. 3). The startup logics involves electrical pre-heating, ignition, certain delays, ramping to operating point etc.

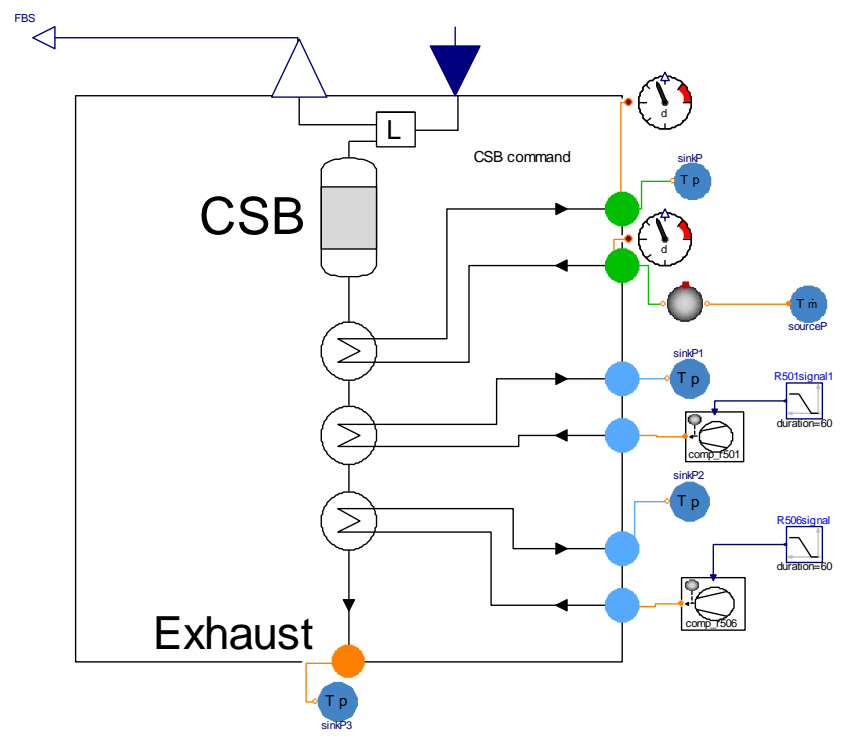

Figure 3: Start system in Dymola

\subsection{Achieving real-time}

A number of model simplifications and changes were introduced to enable real-time simulations:

- Non-linear equations: The submodels were simplified to avoid non-linear equation systems.

- Model simplifications: The model complexity was decreased. The discretized heat exchangers were replaced by lumped models. The discretized ATR, WGS, PROX and CAB reactors were described by lumped reaction volumes connected to lumped heat exchangers.

- Event elimination: The Modelica operator noEvent() was added for applicable functions. Functions not supporting noEvent were exchanged by functions supporting or including noEvent(). In particular IF constructs were removed.

- Minimum flow values: In the real system zero flow or back flow may occur. This can lead to division by zero or very fast dynamics. To avoid this, a negligibly small mass flow is initiated where the real system has zero flow.

- Chemical time scale: The time scale of chemical reactions is generally several orders of magnitude smaller than the required model time step size. Hence the complex dynamic reaction system is replaced by static equations for most reactors. For reactors with dynamic time scales close to the model time step size the chemical reaction rates are damped sufficiently.

- Time scale for flow: Pressure waves travel fast and yield short time scales. To increase the time scales flow losses and volumes were lumped and increased.

- Simplified media: The higher order original NASA Glenn correlations [5] for thermodynamic properties were replaced by linear correlations for the full temperature range. Hence the possible discontinuity by the break temperature is removed.

To enable offline testing of the fuel processor a simple control system was implemented in Dymola and connected to the bus signals in the fuel processor model. An overview of the control system is shown in Fig. 4. Ramps and tables were used for opening and closing of valves and changing set points for mass flow regulators. Simple integrator controllers were used. The purpose of the model control system is only to test the robustness of the model and the model response; hence it is kept simple.

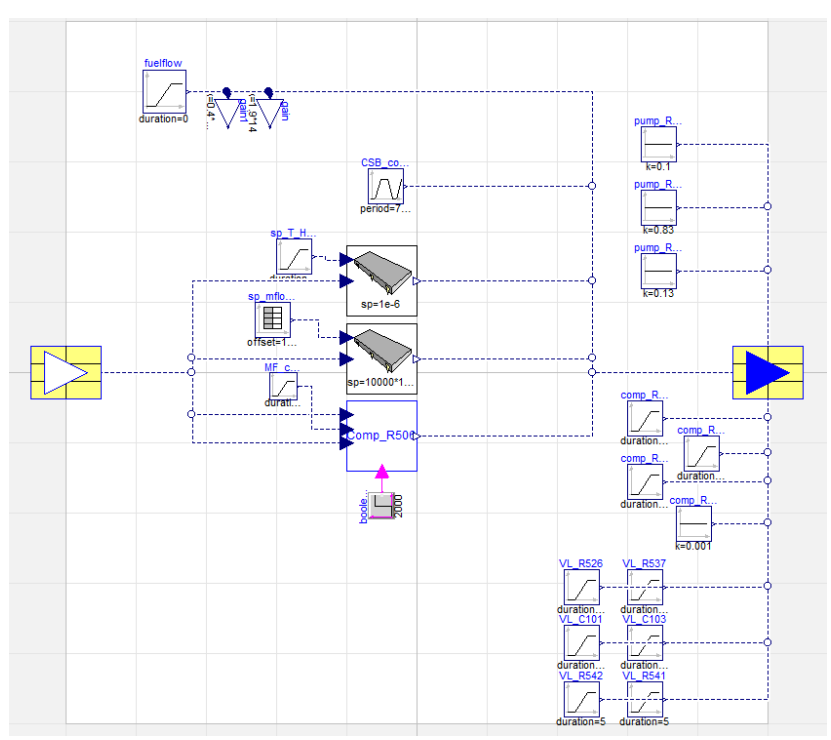

Figure 4: Model control system

\subsection{HIL setup}

The HIL set-up comprises 5 units. The HIL core form the Host computer, where the model is put together and compiled and the Real-time computer, which actually runs the model simulation in real time. The Real-time computer is connected to the PLC running the FCGEN APU control system via CAN buses. For 
operation monitoring and adjustment of control system parameters another computer is used running the SCADA-HMI. This computer can be accessed remotely from J. Stefan Institute, allowing frequent checks and test execution without additional costs. The scheme of the HIL set-up is presented in Fig. 5.

The Real-time computer is a Speedgoat performance real-time target machine running XPC Target. The Dymola Simulink interface is used to import and build the Modelica model in Simulink.
The communication to the PLC includes more than 100 sensor and actuator signals. All signals are added to the model and connected in Simulink. An overview of the model setup in Simulink is seen in Fig. 6.

The time step of the model is $25 \mathrm{~ms}$, which is a tradeoff between model accuracy and the real time requirement. The sample time is $500 \mathrm{~ms}$ to mimic the real system response times. The communication interval to the PLC is $1 \mathrm{~ms}$ to ensure sufficient signal transfer rate.

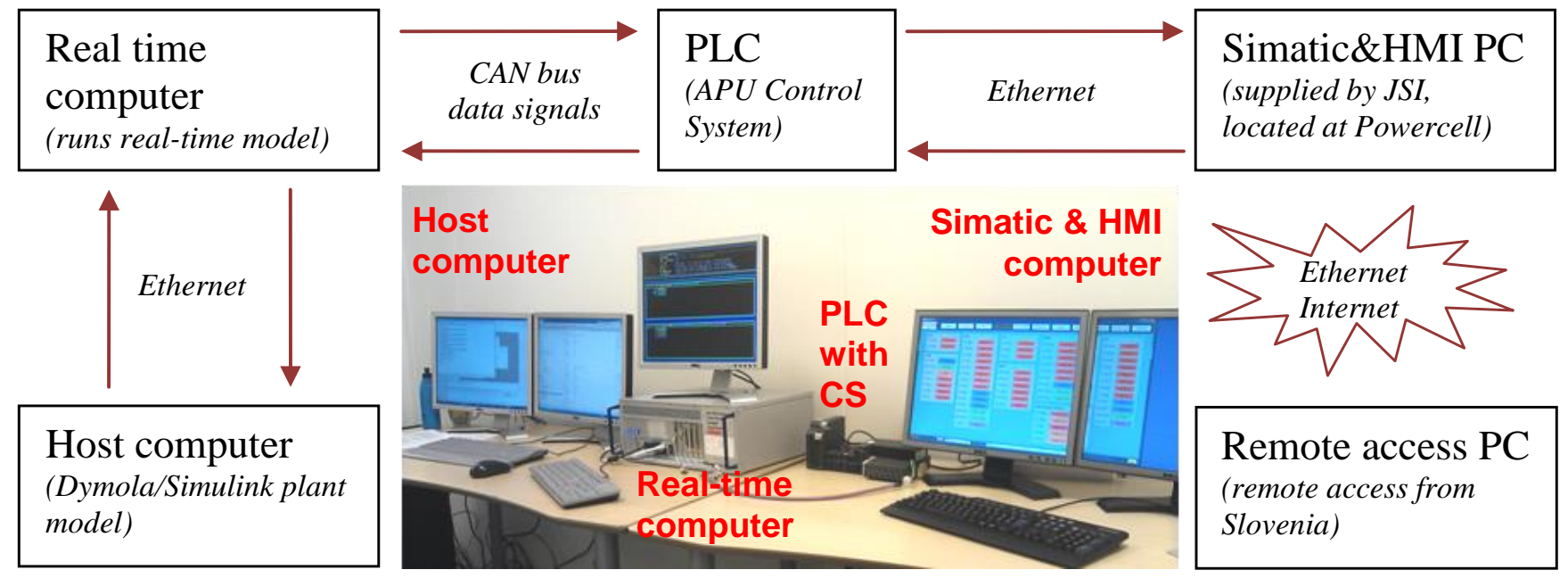

Figure 5: HIL testing assembly scheme

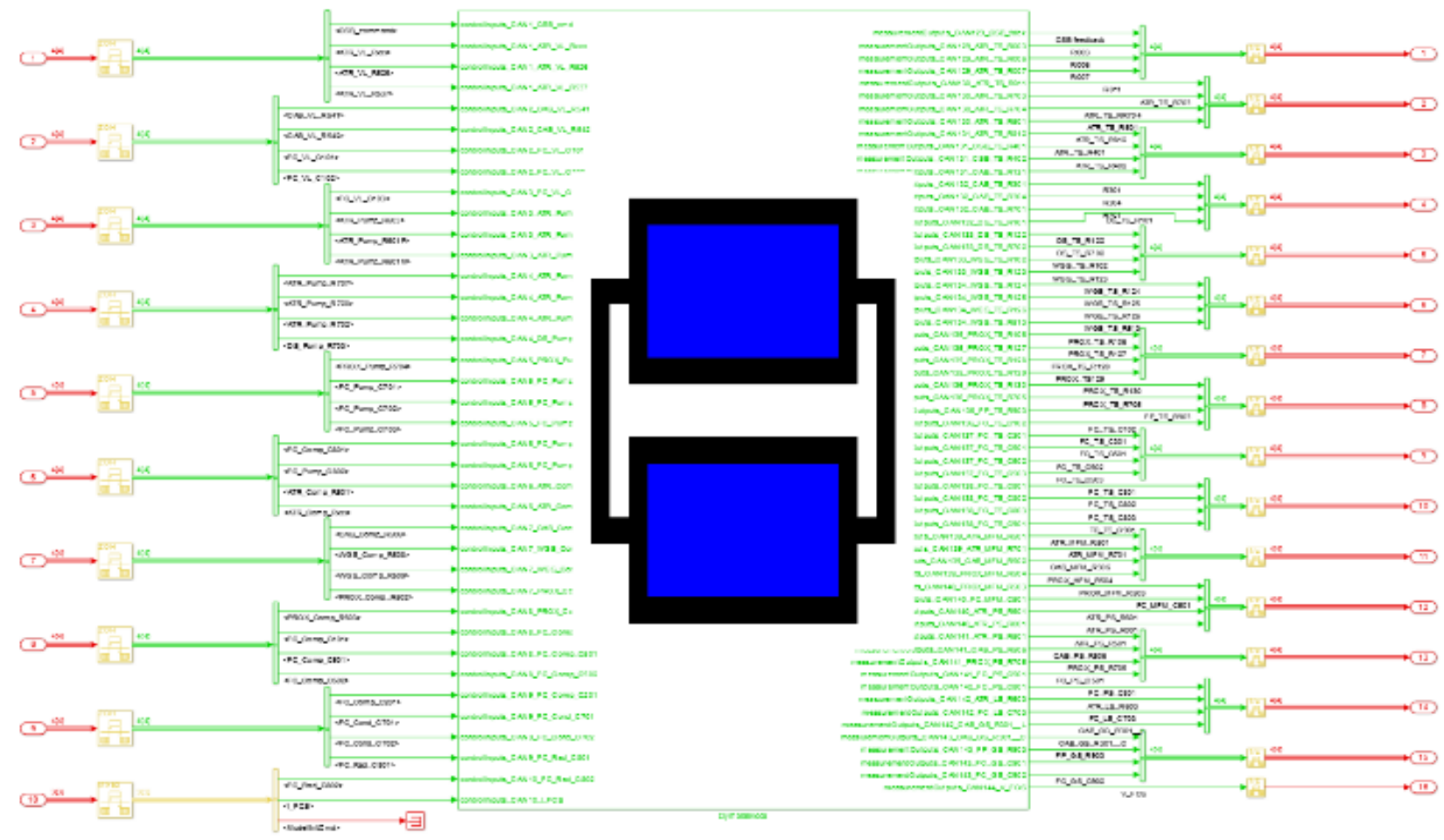

Figure 6: Simulink implementation using the Dymola Simulink interface 


\section{Results and Discussion}

The model is tested offline for phase 1 to 4 in the startup strategy (see section 2.1). Air-fuel ratio and steam mass flow is changed to verify the model response. Following the discussion in section 3, the model cannot be expected to deliver correct absolute values or times. Hence temperatures and times are normalized, and absolute values are not presented.

Temperatures through the system are shown in Fig. 7 and 8. Fig. 7 shows the inlet temperatures to the ATR, Fig. 8 shows the reactor exhaust temperatures through the system. The startup events are indicated on the $\mathrm{x}$ axis.

The overall dynamics of the model is reasonable; inlet air and steam are heated by the startburner, and the temperature drops when the startburner is turned off. The ATR temperature is increased by startburner heating. By ignition, the temperature is increased, and when CSB is turned off the temperature drops to a stable value.

The transient overshoots seen in the figures arise from the use of simple integrator controllers.

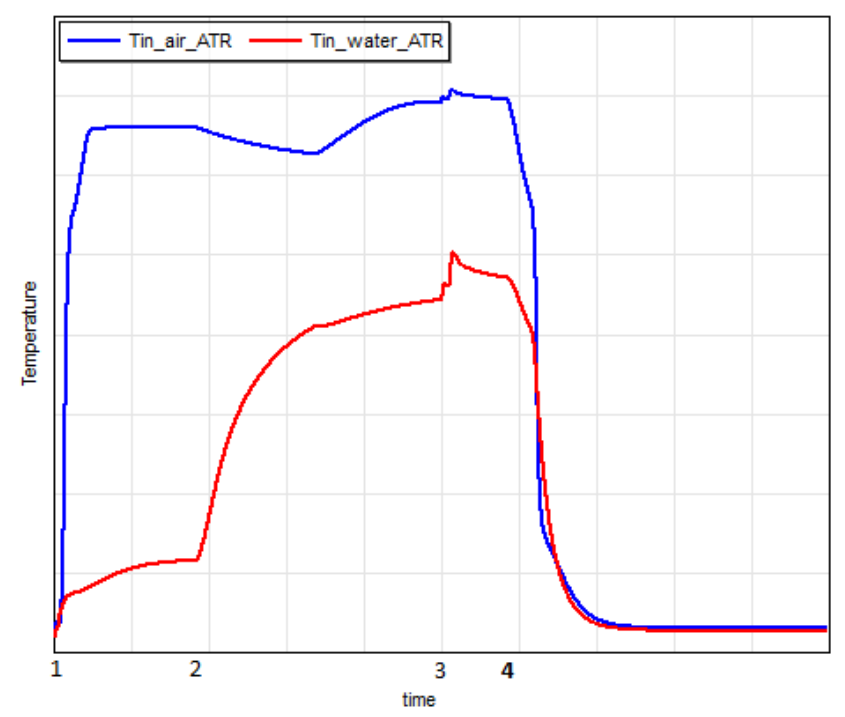

Figure 7: ATR inlet temperatures. The numbers below the $x$ axis indicate start of phase 1-4 defined in section 2.1.

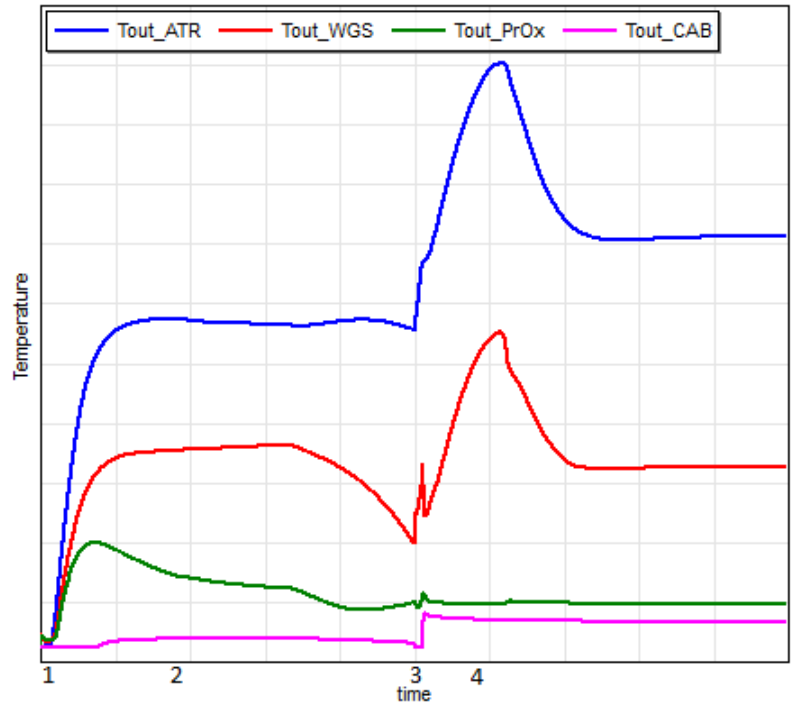

Figure 8: Reactor exhaust temperature. The numbers below the $x$ axis indicate start of phase 1-4 defined in section 2.1.

To test the model response the air-fuel ratio was decreased by lowering the inlet air flow. Since the ATR is run under fuel rich conditions this should lead to decreased ATR outlet temperature. This is confirmed by Fig. 9, where ATR exhaust temperatures for different conditions are plotted.

From this figure it is also confirmed that the ATR temperature is decreased when increasing steam mass flow, as expected.

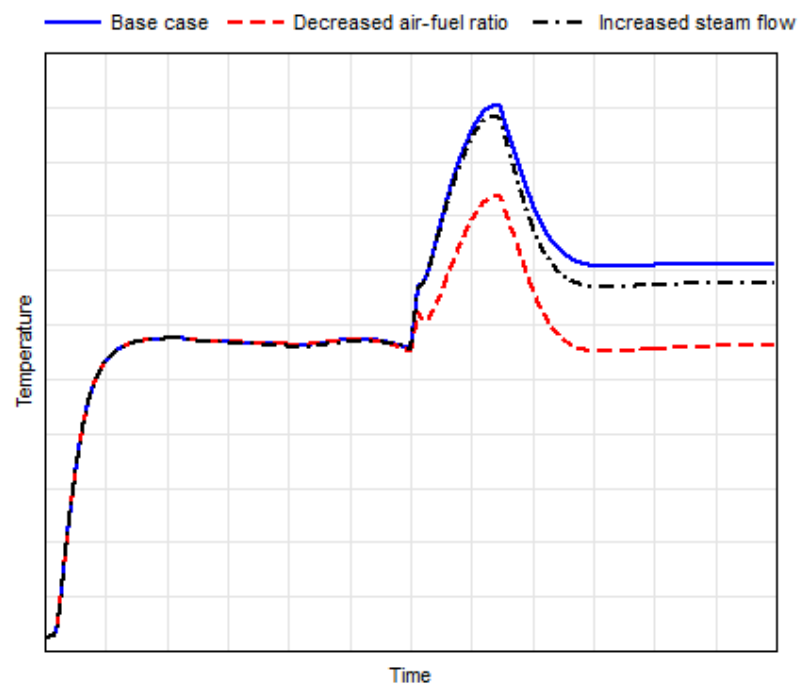

Figure 9: ATR exhaust temperature for different conditions

To meet the real time requirement reactor volumes and flow losses were increased. To evaluate the effect of these necessary changes comparisons of results with and without these changes are shown in Fig. 10 and 11 below. Both simulations were run with the 
Dassl solver. The deviation between original and real time model is considered acceptable.

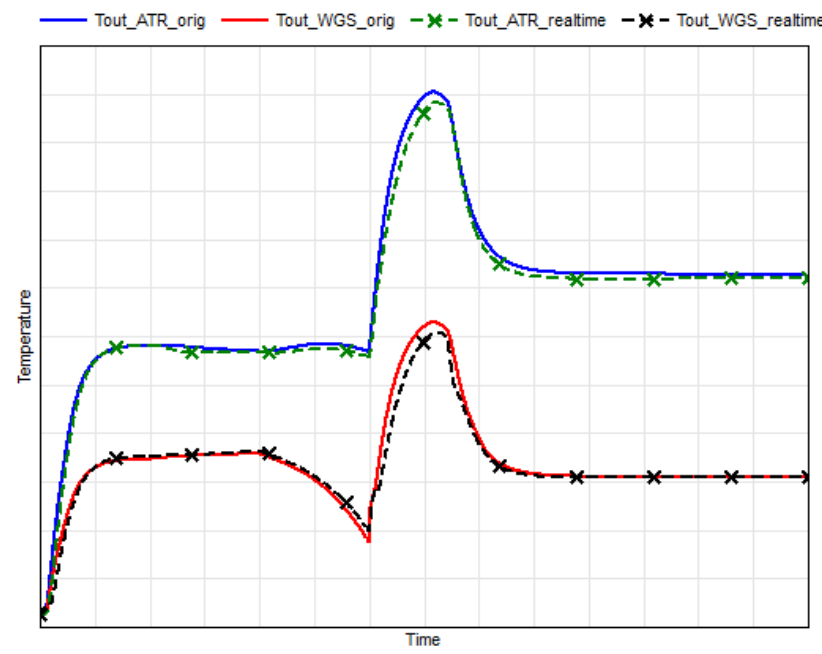

Figure 10: Temperatures before (*_orig) and after (*_realtime) modifications of volumes and flow losses to eliminate time constants below $25 \mathrm{~ms}$.

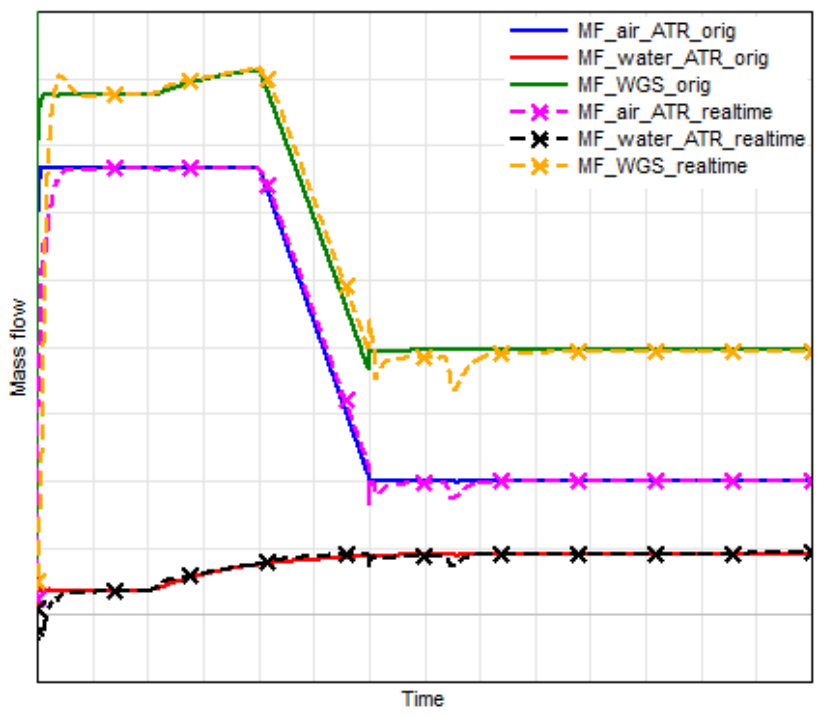

Figure 11: Mass flows before (*_orig) and after (*_realtime) modifications of volumes and flow losses to eliminate time constants below $25 \mathrm{~ms}$.

Using DASSL on a laptop with an Intel Core ${ }^{\mathrm{TM}}$ i73740QM CPU @ $2.70 \mathrm{GHz}$, the model is 68 times faster than real-time. With the Euler solver each time step takes about $4.5 \mathrm{~ms}$ without overruns; the model is 5.6 times faster than realtime when using a time step size of $25 \mathrm{~ms}$. The results are confirmed to be consistent using DASSL and Euler, see Fig. 12. At the Speedgoat machine each time step requires slightly more than $20 \mathrm{~ms}$. For this reason a $25 \mathrm{~ms}$ step size is chosen for the HIL simulations.

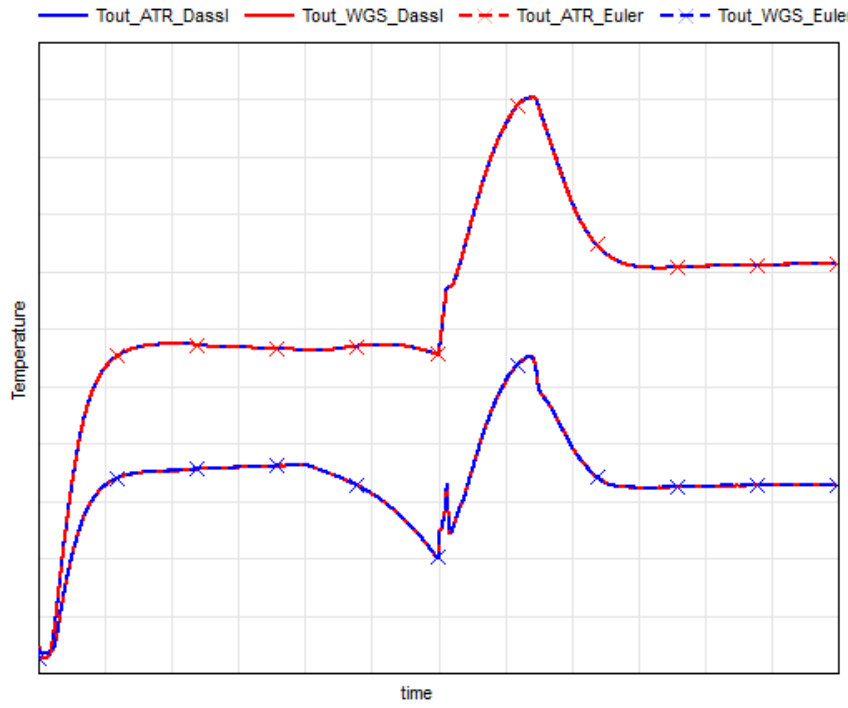

Figure 12: Simulations with DASSL and Euler Solver

The major difficulty in achieving real-time capability was to ensure large enough time constants under all conditions. Lumping volumes in reactors and heat exchangers was not enough. The smallest time constants of the lumped system were $<0.05 \mathrm{~ms}$. To increase the time constants the volumes were increased until the results started to deviate from the original results. Since the time constants were still too small, it was decided to also increase flow losses through the system. This increased the time constants sufficiently while still keeping sufficient accuracy in temperature and mass flows (see Fig. 10 and 11).

The second major difficulty was to ensure stable operation under all circumstances. This was handled by extensive testing of the model, eliminating issues one by one. The major difficulties were connected to back flow and negative mass fractions. Backflow is difficult to consistently handle under all circumstances without event generation and without obtaining nonlinear equation systems. Hence a large enough minimum flow from the compressors, pumps and valves was imposed. Negative mass fractions occur when the solver takes a too large step size for a dynamic reaction where one of the reactants is completely consumed. Hence reactions were limited and max limiters were applied on species mass fractions.

The model is currently tested on the SpeedGoat machine using xPC Target. It is confirmed to successfully load and start. Full HIL tests with a PLC will be the subject of future work. Additional model revisions may be required during the course of the PLC tests. 


\section{Conclusions}

A real-time model for dynamic simulation of a fuel processor is developed. The following main conclusions are made:

- It is possible to create a real-time capable model using Modelica and Dymola for such a fuel processor system.

- A system of 217 dynamical states needs Euler time steps of about $25 \mathrm{~ms}$ to enable HIL simulation on the current hardware configuration.

- Several simplifications were needed to increase the time constants to $25 \mathrm{~ms}$.

\section{Acknowledgements}

This project has received funding from the European Union's Seventh Framework Programme (FP7/20072013) for the Fuel Cells and Hydrogen Joint Technology Initiative under grant agreement $n^{\circ}$ [277844].

\section{References}

[1] FCGEN is financially supported by the European Union's Seventh Framework Programme (FP7/2007-2013) for the Fuel Cells and Hydrogen Joint Technology Initiative under grant agreement $n^{\circ}$ [277844]

[2] Gäfvert, M., Tummescheit, H., Wikander, H., Skoglund, T., Windahl, J., Reutersvärd, P., "Real-Time HWIL simulation of Liquid Food Processor Lines", Modelica conference 2008

[3] FuelCellLib by Modelon, www.modelon.com/products/modelica-libraries/fuel-cell-library/

[4] Andersson, D., Åberg, E., Eborn, J., Yuan, J., Sundén, B., "Dynamic modeling of a solid oxide fuel cell system in Modelica", Modelica conference 2011

[5] McBride, B.J., Zehe, M.J., Gordon, S., NASA Glenn Coefficients for Calculating Thermodynamic Properties of Individual Species, NASA/TP-2002-211556 